\title{
ОБ ОДНОМ МЕТОДЕ РЕГУЛЯРИЗАЦИИ ДЛЯ НЕСОБСТВЕННЫХ ЗАДАЧ ЛИНЕЙНОГО ПРОГРАММИРОВАНИЯ ${ }^{1}$
}

\begin{abstract}
Л. Д. Попов
Продолжено исследование альтернативных схем формирования двойственности в линейном программировании, в основе которых лежит симметричная регуляризация функции Лагранжа одновременно по прямым и двойственным переменным. Особенностью данной работы является использование неевклидовых норм-стабилизаторов. Для новых схем получены симметричные оценки точности получаемого решения. Также проведено исследование свойств метода для случая, когда система ограничений у исходной задачи противоречива. Для таких задач (несобственных 1-го рода) метод дает их обобщенное решение, имеющее хорошую содержательную интерпретацию. Для несобственного случая также получены аналогичные оценки уклонения регуляризированного решения от обобщенного.

Ключевые слова: линейное программирование, двойственность, методы регуляризации, точность решения.

L. D. Popov. On a regularization method for improper linear programs.

We continue the study of alternative duality formation schemes in linear programming based on the symmetric regularization of the Lagrange function simultaneously in the primal and dual variables. A feature of this work is the use of non-Euclidean stabilizing norms. Symmetric bounds for the error of the resulting solution are obtained for the new schemes. The properties of the method are investigated in the case where the constraint system of the original problem is inconsistent. For such problems (improper problems of the first kind), the method gives their generalized solution with an appropriate interpretation. For the improper case, we derive similar estimates for the deviation of the regularized solution from the generalized solution.
\end{abstract}

Keywords: linear programming, duality, regularization methods, accuracy of the solution.

MSC: 90C05, 90C46

DOI: $10.21538 / 0134-4889-2019-25-1-196-206$

\section{Введение}

Пусть имеется пара взаимодвойственных задач ЛП

$$
\begin{gathered}
\min \{(c, x): A x=b, x \geq 0\}, \\
\max \left\{(b, y): A^{T} y \leq c\right\},
\end{gathered}
$$

где векторы $c \in \mathbb{R}^{n}, b \in \mathbb{R}^{m}$ и матрица $A=\left(a_{i j}\right)_{m \times n}$ заданы, векторы $x \in \mathbb{R}^{n}$ и $y \in \mathbb{R}^{m}$ соответствуют прямым и двойственным переменным, $(\cdot, \cdot)$ обозначает скалярное произведение векторов.

Пусть задачи (1), (2) разрешимы и $\bar{x}$ и $\bar{y}$ - их нормальные (минимальные относительно евклидовой нормы) решения. Как известно, эти векторы (как и любые другие решения задач $(1),(2))$ вместе образуют седловую точку функции Лагранжа $L(x, y)=(c, x)-(y, A x-b)$ относительно области $\mathbb{R}_{+}^{n} \times \mathbb{R}^{m}$, где $\mathbb{R}_{+}^{n}$ - неотрицательный ортант $\mathbb{R}^{n}$.

K сожалению, классическая функция Лагранжа с вычислительной точки зрения имеет ряд недостатков, и при разработке численных методов поиска ее седловых точек ее часто заменяют теми или иными ее модификациями. В этой области можно отметить методы обобщенной

\footnotetext{
${ }^{1}$ Исследования поддержаны Российским научным фондом, грант № 14-11-00109.
} 
невязки и регуляризации [1;2], методы проксимальных отображений [3] и модифицированных функций Лагранжа [4;5], методы логарифмических [6] и точных штрафных функций [7-9] и др. В частности, в работах [10-12] для задачи выпуклого программирования исследовалась симметричная регуляризация функции Лагранжа по Тихонову, т. е. регуляризация, проводимая одновременно по прямым и двойственным переменным. Соответствующая расширенная функция Лагранжа имела вид

$$
L^{\sigma}(x, y)=(c, x)-(y, A x-b)+\alpha\|x\|^{2}-\beta\|y\|^{2},
$$

здесь $\alpha>0, \beta>0, \sigma=[\alpha, \beta] \rightarrow 0$ - векторный параметр регуляризации, обе нормы евклидовы.

В упомянутых работах были найдены условия сходимости компонент седловых точек симметрично регуляризированной функции к оптимальным множествам прямой и двойственной задач соответственно (для несобственных задач - к их обобщенным решениям), а для случая, когда параметры регуляризации имеют разный порядок малости, были получены асимметричные оценки уклонения одной из этих компонент от нормального (минимального относительно евклидовой нормы) обычного или обобщенного решения соответствующей задачи. Позднее удалось показать, что в случае задачи линейного программирования (ЛП) при точно заданных исходных данных метод симметричной регуляризации сходится к нормальным решениям по прямым и двойственным переменным одновременно, причем для этого не важно имеют ли параметры регуляризации разные или один порядок малости [13]. Более того, удалось построить более точные и эффективные по сравнению с нелинейным случаем симметричные оценки точности получаемых решений. Эти оценки работают вне зависимости от соотношения между параметрами регуляризации. Данная работа продолжает эти исследования и переносит полученные ранее результаты на более широкий класс стабилизаторов.

\section{1. Альтернативные варианты регуляризации}

Свяжем теперь с исходной парой задач (1), (2) регуляризованную функцию Лагранжа из чуть более широкого класса

$$
L^{\sigma}(x, y)=(c, x)-(y, A x-b)+\alpha\|x\|_{X}^{2}-\beta\|y\|_{Y}^{2},
$$

где $\|x\|_{X}=\left(x^{T} P x\right)^{1 / 2}$ и $\|y\|_{Y}=\left(y^{T} Q y\right)^{1 / 2}-$ монотонные векторные нормы, порождаемые симметричными положительно определенными матрицами $P$ и $Q$.

Будучи сильно выпуклой по одной группе переменных и сильно вогнутой по другой группе, выписанная функция, даже в случае неразрешимости (несобственности) исходных задач, всегда имеет единственную седловую точку относительно области $\mathbb{R}_{+}^{n} \times \mathbb{R}^{m}$. Обозначим эту точку через $\left(x^{\sigma}, y^{\sigma}\right)$. Нас интересует поведение этой точки при различных стратегиях стремления обоих параметров регуляризации к нулю.

Компоненты седловой точки являются решениями прямой и двойственной задач, порождаемых поиском минимакса и максимина функции (3). Первая задача (минимаксная) имеет вид

$$
\min _{x \geq 0} \max _{y} L^{\sigma}(x, y)=\min _{x \geq 0} \Phi^{\sigma}(x)
$$

где

$$
\begin{aligned}
\Phi^{\sigma}(x)= & \max _{y} L^{\sigma}(x, y)=(c, x)+\alpha\|x\|_{X}^{2}+\max _{y}\left\{(y, b-A x)-\beta\|y\|_{Y}^{2}\right\} \\
& =(c, x)+\alpha\|x\|_{X}^{2}+\frac{1}{4 \beta}\|A x-b\|_{Y^{*}}^{2}\left(=L^{\sigma}(x, y(x))\right),
\end{aligned}
$$

здесь $y(x)=(2 \beta)^{-1} Q^{-1}(A x-b),\|z\|_{Y^{*}}=\left(z^{T} Q^{-1} z\right)^{1 / 2}-$ норма, сопряженная к норме $\|\cdot\|_{Y}$. 
Вторая задача (максиминная) записывается как

$$
\max _{y} \min _{x \geq 0} L^{\sigma}(x, y)=\max _{y} \Psi^{\sigma}(y)
$$

где

$$
\begin{aligned}
\Psi^{\sigma}(y) & =\min _{x \geq 0} L^{\sigma}(x, y)=(b, y)-\beta\|y\|_{Y}^{2}+\min _{x \geq 0}\left\{\left(x, c-A^{T} y\right)+\alpha\|x\|_{X}^{2}\right\} \\
= & (b, y)-\beta\|y\|_{Y}^{2}-\frac{1}{4 \alpha}\left\|\left(A^{T} y-c\right)^{+}\right\|_{X^{*}}^{2} \quad\left(=L^{\sigma}(x(y), y)\right) .
\end{aligned}
$$

Здесь $x(y)=(2 \alpha)^{-1} P^{-1}\left(c-A^{T} y\right)^{+} \geq 0, a^{+}=\max \{0, a\},\|z\|_{X^{*}}=\left(z^{T} P^{-1} z\right)^{1 / 2}-$ норма, сопряженная к норме $\|\cdot\|_{X}$.

Мы выведем интересующие нас оценки уклонения получаемых прямых решений от точных, изучая первую из этих задач. Построение двойственных оценок может быть сделано на основе второй задачи по симметричной схеме.

Обсудим далее возможную ситуацию с несовместностью систем ограничений исходных задач, т. е. ситуацию, когда исходные задачи оказались несобственными.

Ограничимся рассмотрением несобственных задач 1-го рода как наиболее важного для практики случая. У таких задач противоречива прямая система ограничений, но совместна двойственная. Содержательно для приведения несобственной задачи 1-го рода к разрешимому виду достаточно всего лишь таким образом скорректировать правые части ее ограничений, чтобы последние оказались совместными. Естественно требовать, чтобы такая корректировка была по возможности минимальной.

Стандартным образом [8] погрузим прямую задачу (1) в параметрическое семейство задач вида

$$
\min \{(c, x): A x=b+u, x \geq 0\}, \quad u \in \mathbb{R}^{m} \text { - параметр. }
$$

Обозначим через $M(u)=\{x: A x=b+u, x \geq 0\}$ допустимые множества этих задач. Множество

$$
\Omega=\{u: M(u) \neq \varnothing\}
$$

называется множеством разрешимости несобственной задачи (1). Очевидно, что оно не пусто и полиэдрально.

Обозначим через $v(u)$ оптимальное значение задачи (5), а через $\bar{x}(u)-$ ее нормальное решение, которое мы определим как

$$
\bar{x}(u)=\arg \min \left\{\|x\|_{X}^{2}: A x=b+u,(c, x) \geq v(u), x \geq 0\right\} .
$$

Как известно, оптимальное значение $v(u)$ задачи (5) является выпуклой, конечной на $\Omega$, непрерывной и даже кусочно-линейной функцией параметра $u$, причем $v(0)=\bar{v}$. Вне множества $\Omega$ будем считать $v(u)=+\infty$. При этом

$$
v(u) \geq \bar{v}+(\bar{y}, u) \geq \bar{v}-\|\bar{y}\|_{Y}\|u\|_{Y^{*}},
$$

поскольку, как известно, $\bar{y}$ является субградиентом функции $v(u)$ в нуле [8].

В свою очередь нормальное решение $\bar{x}(u)$, будучи решением выписанной выше задачи выпуклого программирования (6) с сильно выпуклой целевой функцией, непрерывно зависит от правых частей ее системы ограничений $[14 ; 15]$, в нашем случае от $u$, поскольку $v(u)$ также непрерывна по $u \in \Omega$. Тем самым $\bar{x}(u) \rightarrow \bar{x}(0)=\bar{x}$ при $u \rightarrow+0$.

Введем минимальный относительно сопряженной нормы вектор коррекции правых частей ограничений прямой задачи

$$
\bar{u}=\arg \min _{M(u) \neq \varnothing}\|u\|_{Y^{*}} .
$$

Вектор $\bar{u}$ является метрической проекцией нулевого вектора на выпуклое и полиэдральное множество разрешимости несобственной задачи (1). 
Введем также вектор $\overline{\bar{x}}$ - минимальное относительно нормы $\|\cdot\|_{X}$ решение минимально скорректированной задачи

$$
\min \{(c, x): A x=b+\bar{u}, x \geq 0\},
$$

и вектор $\overline{\bar{y}}$ - минимальное относительно нормы $\|\cdot\|_{Y}$ решение двойственной задачи

$$
\max \left\{(b+\bar{u}, y): A^{T} y \leq c\right\} .
$$

Заметим, что вектор $\overline{\bar{x}}$ может рассматриваться как некоторое обобщенное решение исходной несобственной постановки.

\section{2. Вспомогательные результаты}

Для последующих построений нам необходимо предварительно решить ряд вопросов.

Пусть имеется два выпуклых замкнутых множества $M$ и $N$ в некотором фиксированном пространстве $\mathbb{R}^{s}$. Наряду с евклидовой нормой $\|z\|^{2}=(x, x)$ введем для векторов этого пространства также норму $\|z\|_{R}=z^{T} R z$, порожденную положительно определенной симметричной матрицей $R$. Выпишем разложение по Холецкому матрицы $R=L^{T} L$. Предположим, что множества $M$ и $N$ близки друг к другу в метрике Хаусдорфа

$$
\rho(M, N)=\max \left\{\sup _{x \in M} \inf _{y \in N}\|x-y\|_{R} ; \sup _{y \in N} \inf _{x \in M}\|x-y\|_{R}\right\},
$$

так что $\rho(M, N)<\epsilon, \epsilon>0$ - мало. Покажем, что в этом случае метрические проекции нуля на множества $M, N$ также близки.

Лемма 1. Пусть найденъ метрические проекиии нуля

$$
\begin{gathered}
\bar{z}_{M}=\arg \min _{z \in M}\|z\|_{R} \quad\left(=\arg \min \left\{z^{T} R z: z \in M\right\}\right), \\
\bar{z}_{N}=\arg \min _{z \in N}\|z\|_{R} \quad\left(=\arg \min \left\{z^{T} R z: z \in N\right\}\right)
\end{gathered}
$$

на множества $M$ и $N$ и пусть $\rho(M, N)<\epsilon$. Тогда

$$
\left\|\bar{z}_{N}\right\|_{R}-\epsilon \leq\left\|\bar{z}_{M}\right\|_{R} \leq\left\|\bar{z}_{N}\right\|_{R}+\epsilon, \quad\left\|\bar{z}_{M}\right\|_{R}-\epsilon \leq\left\|\bar{z}_{N}\right\|_{R} \leq\left\|\bar{z}_{M}\right\|_{R}+\epsilon .
$$

Д о к а з а т е л ь с т в о. В самом деле, рассмотрим элементы

$$
\bar{z}_{M}^{\prime}=\arg \min _{z \in M}\left\|z-\bar{z}_{N}\right\|_{R} \in M \quad \text { и } \quad \bar{z}_{N}^{\prime}=\arg \min _{z \in N}\left\|z-\bar{z}_{M}\right\|_{R} \in N .
$$

По условию леммы $\rho(M, N)<\epsilon$, так что $\left\|\bar{z}_{M}^{\prime}-\bar{z}_{N}\right\|_{R}<\epsilon,\left\|\bar{z}_{N}^{\prime}-\bar{z}_{M}\right\|_{R}<\epsilon$. Поэтому в силу определения точек $\bar{z}_{M}$ и $\bar{z}_{N}$ и по неравенству треугольника имеем

$$
\begin{aligned}
& \left\|\bar{z}_{M}\right\|_{R}=\min _{z \in M}\|z\|_{R} \leq\left\|\bar{z}_{M}^{\prime}\right\|_{R} \leq\left\|\bar{z}_{N}\right\|_{R}+\left\|\bar{z}_{N}-\bar{z}_{M}^{\prime}\right\|_{R}<\left\|\bar{z}_{N}\right\|_{R}+\epsilon, \\
& \left\|\bar{z}_{N}\right\|_{R}=\min _{z \in N}\|z\|_{R} \leq\left\|\bar{z}_{N}^{\prime}\right\|_{R} \leq\left\|\bar{z}_{M}\right\|_{R}+\left\|\bar{z}_{M}-\bar{z}_{N}^{\prime}\right\|_{R}<\left\|\bar{z}_{M}\right\|_{R}+\epsilon .
\end{aligned}
$$

Оставшиеся два неравенства получаются из уже установленных перегруппировкой слагаемых:

$$
\left\|\bar{z}_{N}\right\|_{R}>\left\|\bar{z}_{M}\right\|_{R}-\epsilon \quad \text { и } \quad\left\|\bar{z}_{M}\right\|_{R}>\left\|\bar{z}_{N}\right\|_{R}-\epsilon .
$$

Все четыре неравенства установлены.

Вернемся к исходным задачам. Переформулируем ряд вспомогательных оценок, известных для евклидовых норм-стабилизаторов. Рассмотрим вначале разрешимый случай. 
Лемма 2. Пусть исходные задачи разрешимы и $\bar{x} u \bar{y}-$ некоторые их решения, $\bar{v}-u x$ общее оптимальное значение. Пусть такэе $\left(x^{\sigma}, y^{\sigma}\right)-$ седловая точка функиии (3). Тогда выполнены оценки

$$
\begin{gathered}
\bar{v}-\beta\|\bar{y}\|_{Y}^{2} \leq L^{\sigma}\left(x^{\sigma}, y^{\sigma}\right) \leq \bar{v}+\alpha\|\bar{x}\|_{X}^{2}, \\
\left\|A x^{\sigma}-b\right\|_{Y^{*}}^{2} \leq 4 \beta\left[\alpha\|\bar{x}\|_{X}^{2}+\beta\|\bar{y}\|_{Y}^{2}\right]
\end{gathered}
$$

а такље

$$
\bar{v}-\|\bar{y}\|_{Y}\left\|A x^{\sigma}-b\right\|_{Y^{*}} \leq\left(c, x^{\sigma}\right) \leq \bar{v}+\alpha\|\bar{x}\|_{X}^{2} .
$$

Д о к а з а т е л ь с т в о этого утверждения может быть проведено по той же схеме, что использовалась при доказательстве аналогичных утверждений в работах [10-12], где рассматривались евклидовы нормы векторов. При этом вносимые изменения достаточно просты и касаются свойств сопряженных векторных норм. В качестве решений $\bar{x}$ и $\bar{y}$ естественно выбрать решения, минимальные относительно норм $\|\cdot\|_{X}$ и $\|\cdot\|_{Y}$ соответственно..

В неразрешимом случае известные оценки выглядят по-другому.

Лемма 3. Пусть задача (1) - несобственная 1-го рода, и пусть $x^{\sigma}$ - оптимальный вектор задачи (4). Тогда верны оченки:

$$
\begin{gathered}
\left\|A x^{\sigma}-b-\bar{u}\right\|_{Y^{*}} \leq C_{1} \sqrt{\beta} \\
(c, \overline{\bar{x}})-C_{1}\|\overline{\bar{y}}\|_{Y} \sqrt{\beta} \leq\left(c, x^{\sigma}\right) \leq(c, \overline{\bar{x}})+\alpha\|\overline{\bar{x}}\|_{X}^{2},
\end{gathered}
$$

где $C_{1}=2\left[\sqrt{\beta}\|\overline{\bar{y}}\|_{Y}+\sqrt{\alpha\|\overline{\bar{x}}\|_{X}^{2}+\beta\|\overline{\bar{y}}\|_{Y}^{2}}\right]$.

Д о к а з а т е л ь с т в о этого утверждения также может быть проведено по схеме из работ [10-12].

\section{3. Оценки уклонения в разрешимом случае}

Перейдем к основной цели исследования.

Предположим вначале, что исходные задачи разрешимы. Оценим величину $\|\bar{x}(u)-\bar{x}\|_{X}$. Определим оптимальное множество задачи (5) как

$$
\bar{M}(u)=\{x: A x=b+u,(c, x) \leq v(u), x \geq 0\} .
$$

Как видно из (6), вектор $\bar{x}(u)$ является метрической проекцией нуля на оптимальное множество задачи (5) $\bar{M}(u)$, а вектор $\bar{x}$ - метрической проекцией нуля на оптимальное множество задачи (1) $\bar{M}(0)$. Оба эти множества определяются как множества решений двух систем неравенств и уравнений с одними и теми же матрицами коэффициентов, но с разными правыми частями в этих неравенствах. Согласно лемме Хоффмана [16] существует такая константа $K$, зависящая только от матрицы $A$ и вектора $c$, что хаусдорфово расстояние

$$
\rho(\bar{M}(u), \bar{M}(0)) \leq K\left(\|u\|_{Y^{*}}+|v(u)-\bar{v}|\right) \leq K\left(1+\|\bar{y}\|_{Y}\right)\|u\|_{Y^{*}}
$$

(здесь также применено соотношение (7)). Строго говоря, в лемме Хоффмана используются несколько иные векторные нормы (чебышевская и октаэдрическая), но поскольку в конечномерных пространствах все нормы эквивалентны, выше использованы интересующие нас альтернативные нормы (очевидно, это сказывается только на величине используемых констант).

Применяем к сложившейся ситуации лемму 3 . Получаем

Следствие 1. Верны оченки

$$
\|\bar{x}(u)\|_{X} \leq\|\bar{x}\|_{X}+K\left(1+\|\bar{y}\|_{Y}\right)\|u\|_{Y^{*}}, \quad\|\bar{x}\|_{X} \leq\|\bar{x}(u)\|_{X}+K\left(1+\|\bar{y}\|_{Y}\right)\|u\|_{Y^{*}} .
$$


Вернемся к вектору $x^{\sigma}$.

Лемма 4. Верна оченка $\left\|x^{\sigma}\right\|_{X} \leq\|\bar{x}\|_{X}+K\left(1+\|\bar{y}\|_{Y}\right)\left\|A x^{\sigma}-b\right\|_{Y^{*}}$.

Д о к а з а т е л ь с т в о. Положим $u^{\sigma}=A x^{\sigma}-b \in \mathbb{R}^{m}$. Обозначим через $\bar{x}\left(u^{\sigma}\right)$ оптимальный вектор задачи (6) с $u=u^{\sigma}$. По определению вектора $x^{\sigma}$ верно неравенство

$$
\Phi^{\sigma}\left(x^{\sigma}\right)=\min _{x \geq 0} \Phi^{\sigma}(x) \leq \Phi^{\sigma}\left(\bar{x}\left(u^{\sigma}\right)\right),
$$

или, что то же,

$$
\left(c, x^{\sigma}\right)+\frac{1}{4 \beta}\left\|u^{\sigma}\right\|_{Y^{*}}^{2}+\alpha\left\|x^{\sigma}\right\|_{X}^{2} \leq v\left(u^{\sigma}\right)+\frac{1}{4 \beta}\left\|A \bar{x}\left(u^{\sigma}\right)-b\right\|_{Y^{*}}^{2}+\alpha\left\|\bar{x}\left(u^{\sigma}\right)\right\|_{X}^{2} .
$$

Поскольку вектор $x^{\sigma}$ допустим для задачи (6) при $u=u^{\sigma}=A x^{\sigma}-b$, а вектор $\bar{x}\left(u^{\sigma}\right)$ для нее оптимален, то из последнего неравенства после перегруппировки его слагаемых вытекает, что

$$
\alpha\left\|x^{\sigma}\right\|_{X}^{2} \leq \alpha\left\|\bar{x}\left(u^{\sigma}\right)\right\|_{X}^{2}+\frac{1}{4 \beta}[\underbrace{\left.\left\|A \bar{x}\left(u^{\sigma}\right)-b\right\|_{Y^{*}}^{2}-\left\|u^{\sigma}\right\|_{Y^{*}}^{2}\right]}_{=0}+\underbrace{v\left(u^{\sigma}\right)-\left(c, x^{\sigma}\right)}_{\leq 0} \leq \alpha\left\|\bar{x}\left(u^{\sigma}\right)\right\|_{X}^{2},
$$

т. е. $\left\|x^{\sigma}\right\|_{X} \leq\left\|\bar{x}\left(u^{\sigma}\right)\right\|_{X}$, что вкупе со следствием 1 завершает доказательство.

Следствие 2. Верна оценка $\left\|x^{\sigma}\right\|_{X} \leq\|\bar{x}\|_{X}+2 K\left(1+\|\bar{y}\|_{Y}\right) \sqrt{\beta\left[\alpha\|\bar{x}\|_{X}^{2}+\beta\|\bar{y}\|_{Y}^{2}\right]}$.

Здесь было применено свойство (8).

Рассмотрим далее задачу

$$
\min \{(P \bar{x}, x): A x=b,(c, x) \leq \bar{v}, x \geq 0\} .
$$

Она получается в результате линеаризации задачи (6) при $u=0$ в точке $\bar{x}$. Эта же точка $\bar{x}$ будет одним из решений этой задачи и $(P \bar{x}, \bar{x})=\|\bar{x}\|_{X}^{2}$. Разрешима будет и двойственная к ней задача

$$
\max \left\{(b, \lambda)-\bar{v} \lambda_{0}: A^{T} \lambda-\lambda_{0} c \leq P \bar{x}, \lambda_{0} \geq 0\right\},
$$

одно из решений которой обозначим через $\left[\bar{\lambda}, \bar{\lambda}_{0}\right]$.

Лемма 5. Для любого $x \geq 0$ верна оценка $(P \bar{x}, x) \geq\|\bar{x}\|_{X}^{2}-\|\bar{\lambda}\|_{Y}\|A x-b\|_{Y^{*}}-\bar{\lambda}_{0}((c, x)-\bar{v})^{+}$.

Д о к а з а т е л ь с т в о. Внесем параметры в правые части ограничений задачи (13) следующим образом:

$$
\min \{(P \bar{x}, x): A x=b+u,(c, x) \leq \bar{v}+\delta, x \geq 0\}
$$

здесь $\delta>0, u \in \Omega$ - параметры. Как обычно, оценим снизу оптимальные значения $v(u, \delta)$ этих задач через двойственные оценки их ограничений:

$$
v(u, \delta) \geq v(0,0)+(\bar{\lambda}, u)-\bar{\lambda}_{0} \delta \geq\|\bar{x}\|_{X}^{2}-\|\bar{\lambda}\|_{Y}\|u\|_{Y^{*}}-\bar{\lambda}_{0} \delta .
$$

Для завершения доказательства остается заметить, что произвольный вектор $x \geq 0$ будет допустимым для задачи (14) при $u=A x-b$ и $\delta=|(c, x)-\bar{v}|$, так что

$$
(P \bar{x}, x) \geq v(A x-b,|(c, x)-\bar{v}|) \geq\|\bar{x}\|_{X}^{2}-\|\bar{\lambda}\|_{Y}\left\|(A x-b)^{+}\right\|_{Y^{*}}-\bar{\lambda}_{0}|(c, x)-\bar{v}|,
$$

что и требовалось.

Следствие 3. Верна оченка $\left(P \bar{x}, x^{\sigma}\right) \geq\|\bar{x}\|_{X}^{2}-\|\bar{\lambda}\|_{Y} \sqrt{\beta\left[\alpha\|\bar{x}\|_{X}^{2}+\beta\|\bar{y}\|_{Y}^{2}\right]}-\alpha \bar{\lambda}_{0}\|\bar{x}\|_{X}^{2}$. 
Здесь были также применены свойства (8), (9).

Введем следующие константы

$G(a l, \beta)=2 K\left(1+\|\bar{y}\|_{Y}\right) \sqrt{\beta\left[\alpha\|\bar{x}\|_{X}^{2}+\beta\|\bar{y}\|_{Y}^{2}\right]}, \quad H(\alpha, \beta)=\|\bar{\lambda}\|_{Y} \sqrt{\beta\left[\alpha\|\bar{x}\|_{X}^{2}+\beta\|\bar{y}\|_{Y}^{2}\right]}+\alpha \bar{\lambda}_{0}\|\bar{x}\|_{X}^{2}$.

Теорема 1. Верна оценка $\left\|x^{\sigma}-\bar{x}\right\|_{X} \leq \sqrt{2\|\bar{x}\|_{X} G(\alpha, \beta)+G(\alpha, \beta)^{2}+2 H(\alpha, \beta)}$.

Д о к а з а т е л ь с т в о. Следствия 2, 3 утверждают, что

$$
\begin{gathered}
\left\|x^{\sigma}\right\|_{X} \leq\|\bar{x}\|_{X}+2 K\left(1+\|\bar{y}\|_{Y}\right) \sqrt{\beta\left[\alpha\|\bar{x}\|_{X}^{2}+\beta\|\bar{y}\|_{Y}^{2}\right]}=\|\bar{x}\|_{X}+G(\alpha, \beta), \\
\left(P \bar{x}, x^{\sigma}\right) \geq\|\bar{x}\|_{X}^{2}-\|\bar{\lambda}\|_{Y} \sqrt{\beta\left[\alpha\|\bar{x}\|_{X}^{2}+\beta\|\bar{y}\|_{Y}^{2}\right]}-\alpha \bar{\lambda}_{0}\|\bar{x}\|_{X}^{2}=\|\bar{x}\|_{X}^{2}-H(\alpha, \beta) .
\end{gathered}
$$

Отсюда

$$
\begin{gathered}
\left\|x^{\sigma}-\bar{x}\right\|_{X}^{2}=\left(x^{\sigma}-\bar{x}\right)^{T} P\left(x^{\sigma}-\bar{x}\right) \\
=\left\|x^{\sigma}\right\|_{X}^{2}+\|\bar{x}\|_{X}^{2}-2\left(P \bar{x}, x^{\sigma}\right) \leq\left(\|\bar{x}\|_{X}+G(\alpha, \beta)\right)^{2}+\|\bar{x}\|_{X}^{2}-2\left(\|\bar{x}\|_{X}^{2}-H(\alpha, \beta)\right) \\
=2\|\bar{x}\|_{X} G(\alpha, \beta)+G(\alpha, \beta)^{2}+2 H(\alpha, \beta) .
\end{gathered}
$$

Доказательство завершено.

Приведем более грубую, но зато более компактную оценку рассматриваемых уклонений.

Следствие 4. Пусть $\|\sigma\|_{\infty}=\max \{\alpha, \beta\}<1$. Тогда $\left\|x^{\sigma}-\bar{x}\right\|_{X} \leq N_{x}\|\sigma\|_{\infty}^{1 / 2}$ при некотором фиксированном $N_{x}$.

Следствие 5. Пусть $\|\sigma\|_{\infty}=\max \{\alpha, \beta\} \rightarrow+0$. Тогда $x^{\sigma} \rightarrow \bar{x}$.

Вывод этих следствий очевиден.

Заметим далее, что схемы формирования двойственности в теории ЛП носят симметричный характер. Это значит, что для уклонения $\left\|y^{\sigma}-\bar{y}\right\|_{Y}$ можно построить аналогичные оценки (с симметричной перестановкой параметров регуляризации) тем, что уже построены для $\left\|x^{\sigma}-\bar{x}\right\|_{X}$. В виду понятной структуры, но громоздкости этих оценок последние здесь не выписываются. Вместо этого ниже сформулированы аналоги следствий 4, 5 .

Следствие 6. Пусть $\|\sigma\|_{\infty}=\max \{\alpha, \beta\}<1$. Тогда $\left\|y^{\sigma}-\bar{y}\right\|_{Y} \leq N_{y}\|\sigma\|_{\infty}^{1 / 2}$ при некотором фиксированном $N_{y}$.

Следствие 7. Пусть $\|\sigma\|_{\infty}=\max \{\alpha, \beta\} \rightarrow+0$. Тогда $y^{\sigma} \rightarrow \bar{y}$.

Объединяя следствия 5,7 , можно сформулировать завершающее утверждение.

Теорема 2. Пусть $\|\sigma\|_{\infty}=\max \{\alpha, \beta\} \rightarrow+0$. Тогда $x^{\sigma} \rightarrow \bar{x} u y^{\sigma} \rightarrow \bar{y}$.

Таким образом в линейном случае имеет место одновременная сходимость компонент седловой точки функции (3) к нормальным решениям прямой и двойственной задач и нет необходимости делать один из параметров регуляризации бесконечно малой более высокого порядка малости, чем другой.

П р и м е ч а н и е. Сравним по точности полученные выше оценки и оценки, полученные ранее в работах [10-12] для задач линейного и выпуклого программирования для евклидовых норм. Рассмотрим только прямые переменные. Поскольку более ранняя оценка имела вид

$$
\left\|x^{\sigma}-\bar{x}\right\| \leq 1 / 2\left[\|\bar{y}\| \sqrt{\frac{\beta}{\alpha}}+\sqrt{\alpha \beta}\left\|\left[\bar{\lambda}, \bar{\lambda}_{0}\right]\right\|\right]
$$


то она полезна только при $\beta=o(\alpha)$. Поэтому введем две бесконечно малые положительные величины $\alpha$ и $\epsilon$ и положим $\beta=\alpha \epsilon$. Подставляя эти формулы в правую часть последнего неравенства, получаем, что $R H S_{1}=1 / 2\left[\|\bar{y}\| \sqrt{\beta / \alpha}+\sqrt{\alpha \beta}\left\|\left[\bar{\lambda}, \bar{\lambda}_{0}\right]\right\|\right] \sim O\left(\epsilon^{1 / 2}\right)$. Если же подставить их в правую часть оценки из теоремы 2 и учесть, что $G(\alpha, \alpha \epsilon) \sim O\left(\alpha \epsilon^{1 / 2}\right)$, а $H(\alpha, \alpha \epsilon) \sim O(\alpha)$, то для евклидовых норм имеем

$$
R H S_{2}=\sqrt{2\|\bar{x}\| G(\alpha, \beta)+G(\alpha, \beta)^{2}+2 H(\alpha, \beta)} \sim O\left(\alpha^{1 / 2}\right) .
$$

Таким образом, вновь полученная оценка оказывается точнее известной за исключением лишь тех случаев, когда параметр $\beta$ является бесконечно малой более высокого порядка, чем $\alpha^{2}$. Кроме того, она сохраняет свою полезность и тогда, когда $\alpha$ и $\beta$ имеют одинаковый порядок малости или даже $\alpha$ имеет порядок малости, больший чем $\beta$.

\section{4. Оценки для несобственных задач 1-го рода}

Рассмотрим теперь случай, когда ограничения прямой задачи (1) противоречивы, в то время как двойственная задача (2) имеет хотя бы один допустимый вектор.

Вновь обратимся к задаче (4)

$$
\min _{x \geq 0} \max _{y} L^{\sigma}(x, y)=\min _{x \geq 0} \Phi^{\sigma}(x),
$$

где $\Phi_{\sigma}(x)=\max _{y} L^{\sigma}(x, y)=(c, x)+\frac{1}{4 \beta}\|A x-b\|_{Y^{*}}^{2}+\alpha\|x\|_{X}^{2}$. Несмотря на сделанные предположения о противоречивости ограничений исходной задачи (1), выписанная минимаксная задача разрешима и имеет единственное решение. Как и прежде, обозначим это решение через $x^{\sigma}$. Согласно лемме 3

$$
\left\|A x^{\sigma}-b-\bar{u}\right\|_{Y^{*}} \leq C_{1} \sqrt{\beta}, \quad(c, \overline{\bar{x}})-C_{1}\|\overline{\bar{y}}\|_{Y} \sqrt{\beta} \leq\left(c, x^{\sigma}\right) \leq(c, \overline{\bar{x}})+\alpha\|\overline{\bar{x}}\|_{X}^{2},
$$

где $C_{1}=2\left[\sqrt{\beta}\|\overline{\bar{y}}\|_{Y}+\sqrt{\alpha\|\overline{\bar{x}}\|_{X}^{2}+\beta\|\overline{\bar{y}}\|_{Y}^{2}}\right]$.

Попробуем выстроить оценку уклонения $\left\|x^{\sigma}-\overline{\bar{x}}\right\|$.

Подчеркнем, что в случае несобственности 1-го рода последовательность $y^{\sigma}$ будет всегда неограниченной. Повторим с небольшими изменениями общую канву предыдущих рассуждений.

Рассмотрим задачу (6) дважды: один раз с параметром $u=u^{\sigma}=A x^{\sigma}-b$ и второй раз с параметром $u=\bar{u}$. По свойствам двойственных оценок оптимальные значения этих задач связаны неравенством $v(\bar{u})-v\left(u^{\sigma}\right) \leq\left(\overline{\bar{y}}, \bar{u}-u^{\sigma}\right) \leq\|\overline{\bar{y}}\|_{Y}\left\|u^{\sigma}-\bar{u}\right\|_{Y^{*}}$. Поэтому по уже упоминавшейся лемме Хоффмана имеем оценку

$$
\rho\left(\bar{M}\left(u^{\sigma}\right), \bar{M}(\bar{u})\right) \leq K\left(\left\|u^{\sigma}-\bar{u}\right\|_{Y^{*}}+\left|v\left(u^{\sigma}\right)-v(\bar{u})\right|\right) \leq K\left(1+\|\overline{\bar{y}}\|_{Y}\right)\left\|A x^{\sigma}-b-\bar{u}\right\|_{Y^{*}}
$$

для расстояния по Хаусдорфу между оптимальными множествами $\bar{M}\left(u^{\sigma}\right)$ и $\bar{M}(\bar{u})$ этих задач. Комбинируя это неравенство с оценкой (10) и привлекая лемму 1 , получаем следствие.

Следствие 8. Верна оценка $\left\|\bar{x}\left(u^{\sigma}\right)\right\|_{X} \leq\|\bar{x}(\bar{u})\|_{X}+K\left(1+\|\overline{\bar{y}}\|_{Y}\right) C_{1} \sqrt{\beta}$.

Далее выводим аналог леммы 4.

Лемма 6. Верна оценка $\left\|x^{\sigma}\right\|_{X} \leq\|\bar{x}(\bar{u})\|_{X}+K\left(1+\|\overline{\bar{y}}\|_{Y}\right) C_{1} \sqrt{\beta}$.

Д о к а з а т е л ь с т в о. Пусть $\bar{x}\left(u^{\sigma}\right)$ - оптимальный вектор задачи (12), отвечающий параметру $u=u^{\sigma}=A x^{\sigma}-b$. По определению этот вектор удовлетворяет неравенству

$$
\Phi^{\sigma}\left(x^{\sigma}\right)=\min _{x \geq 0} \Phi^{\sigma}(x) \leq \Phi^{\sigma}\left(\bar{x}\left(u^{\sigma}\right)\right),
$$


T. e.

$$
\left(c, x^{\sigma}\right)+\frac{1}{4 \beta}\left\|u^{\sigma}\right\|_{Y^{*}}^{2}+\alpha\left\|x^{\sigma}\right\|_{X}^{2} \leq v\left(u^{\sigma}\right)+\frac{1}{4 \beta}\left\|A \bar{x}\left(u^{\sigma}\right)-b\right\|_{Y^{*}}^{2}+\alpha\left\|\bar{x}\left(u^{\sigma}\right)\right\|_{X}^{2} .
$$

Поскольку $\left\|u^{\sigma}\right\|_{Y^{*}}=\left\|A \bar{x}\left(u^{\sigma}\right)-b\right\|_{Y^{*}}$ и $v\left(u^{\sigma}\right) \leq\left(c, x^{\sigma}\right)$, то после перегруппировки слагаемых имеем

$$
\alpha\left\|x^{\sigma}\right\|_{X}^{2} \leq \alpha\left\|\bar{x}\left(u^{\sigma}\right)\right\|_{X}^{2}+\frac{1}{4 \beta}\left\|A \bar{x}\left(u^{\sigma}\right)-b\right\|_{Y^{*}}^{2}-\frac{1}{4 \beta}\left\|u^{\sigma}\right\|_{Y^{*}}^{2}+v\left(u^{\sigma}\right)-\left(c, x^{\sigma}\right) \leq \alpha\left\|\bar{x}\left(u^{\sigma}\right)\right\|_{X}^{2} .
$$

Тем самым, получено, что $\left\|x^{\sigma}\right\|_{X} \leq\left\|\bar{x}\left(u^{\sigma}\right)\right\|_{X}$; это вкупе со следствием 1 завершает доказательство.

Вернемся к вектору $\overline{\bar{x}}=\arg \min \left\{\|x\|_{X}^{2}: A x=b+\bar{u},(c, x) \leq v(\bar{u}), x \geq 0\right\}$. Линеаризируя эту задачу выпуклого программирования в точке $\overline{\bar{x}}$, приходим к задаче ЛП

$$
\min \{(P \overline{\bar{x}}, x): A x=b+\bar{u},(c, x) \leq v(\bar{u}), x \geq 0\} .
$$

Одним из ее решений будет вектор $\overline{\bar{x}}$. Выпишем также двойственную к ней задачу ЛП

$$
\max \left\{(b+\bar{u}, \lambda)-v(\bar{u}) \lambda_{0}: A^{T} \lambda-\lambda_{0} c \leq P \overline{\bar{x}}, \lambda_{0} \geq 0\right\}
$$

и обозначим через $\bar{w}=\left[\overline{\bar{\lambda}}_{0}, \overline{\bar{\lambda}}\right]$ одно из ее решений. По аналогии с леммой 5 легко вывести следующее утверждение.

Лемма 7. Для любого $x \geq 0$ верна оценка

$$
(P \overline{\bar{x}}, x) \geq\|\overline{\bar{x}}\|_{X}^{2}-\|\overline{\bar{\lambda}}\|_{Y}\|A x-b-\bar{u}\|_{Y^{*}}-\overline{\bar{\lambda}}_{0}|v(\bar{u})-(c, x)| .
$$

Привлекая свойства (10), (11), можно сформулировать следствие.

Следствие 9. Верна оценка $\left(P \overline{\bar{x}}, x^{\sigma}\right) \geq\|\overline{\bar{x}}\|_{X}^{2}-C_{1} \sqrt{\beta}\|\overline{\bar{\lambda}}\|_{Y}-\alpha \overline{\bar{\lambda}}_{0}\|\overline{\bar{x}}\|_{X}^{2}$.

Приведенные лемма 6 и следствие 9 позволяют провести рассуждения, аналогичные тем, что были проведены при доказательстве теоремы 1. Тем самым оказывается доказанным следующее утверждение.

Теорема 3. Верно неравенство

$$
\left\|x^{\sigma}-\overline{\bar{x}}\right\|_{X} \leq \sqrt{2\|\overline{\bar{x}}\|_{X} \cdot G^{\prime}(\alpha, \beta)+G^{\prime}(\alpha, \beta)^{2}+2 H^{\prime}(\alpha, \beta)},
$$

где $G^{\prime}(\alpha, \beta)=2 K\left(1+\|\overline{\bar{y}}\|_{Y}\right) C_{1} \sqrt{\beta}, H^{\prime}(\alpha, \beta)=C_{1} \sqrt{\beta}\|\overline{\bar{\lambda}}\|_{Y}+\alpha \overline{\bar{\lambda}} \| \overline{\bar{x}}_{X}^{2}$.

Завершим раздел упрощенной оценкой.

Следствие 10. Пусть $\|\sigma\|_{\infty}=\max \{\alpha, \beta\}<1$ и задача (1) несобственная 1-го рода. Тогда $\left\|x^{\sigma}-\overline{\bar{x}}\right\|_{X} \leq N_{x}\|\sigma\|_{\infty}^{1 / 2}$ при некотором фиксированном $N_{x}$.

\section{Заключение}

В работе продолжено изучение ставших классическими схем формирования двойственности в линейном программировании, в основе которых лежит симметричная регуляризация функции Лагранжа одновременно по прямым и двойственным переменным. При этом рассмотрен более широкий класс норм-стабилизаторов. Установлено, что в отличие от более ранних результатов для нелинейного (выпуклого) случая метод сходится к нормальным (минимальным относительно нормы-стабилизатора) решениям исходных задач одновременно по прямым и двойственным переменным, причем для этого не важно имеют ли параметры регуляризации разные или один порядок малости. Более того, приведены более эффективные по сравнению с нелинейным случаем симметричные оценки точности получаемых решений. Для несобственных задач ЛП первого рода и их обобщенных решений получены аналогичные оценки по прямым переменным. 


\section{СПИСОК ЛИТЕРАТУРЫ}

1. Тихонов А. Н., Арсенин В. Я. Методы решения некорректных задач. М.: Наука, 1979. 285 с.

2. Васильев Ф. П. Методы оптимизации: в 2 кн. Москва: МЦНМО, 2011. Кн. 1: 620 с.; кн. 2: 433 с.

3. Rockafellar R. T. Augmented lagrange multiplier functions and duality in nonconvex programming // SIAM J. Contr. 1974. Vol. 12, no. 2. P. 268-285. doi: 10.1137/0312021.

4. Evtushenko Ju. Numerical optimization technique. Optimization Software. N Y: Inc. Publ. Division, 1985. 562 p.

5. Гольштейн Е.Г., Третьяков Н.В. Модифицированные функции Лагранжа. Теория и методы оптимизации. Москва: Наука. Главная редакция физ.-мат. литературы, 1989. 400 с.

6. Roos C., Terlaky T. Theory and algorithms for linear optimization: An interior point approach. N Y: Wiley, 1997. $454 \mathrm{p}$.

7. Еремин И. И. Двойственность в линейной оптимизации. Екатеринбург: Изд-во УрО РАН, 2001. $180 \mathrm{c}$.

8. Еремин И. И., Мазуров Вл. Д., Астафьев Н. Н. Несобственные задачи линейного и выпуклого программирования. М.: Наука, 1983. 336 с.

9. Eremin I. I. Theory of linear optimization. Inverse and ill-posed problems series. Utrecht; Boston; Koln; Tokyo: VSP, 2002. 248 p.

10. Скарин В.Д. Об оптимальной коррекции противоречивых задач выпуклого программирования // Тр. Ин-та математики и механики УрО РАН. 2013. Т. 19, № 2. С. 267-274.

11. Skarin V.D. Regularization of the min-max problems occurring in convex programming // Comput. Math. Math. Physics. 1977. Vol. 17, no. 6. P. 65-78. doi: 10.1016/0041-5553(77)90173-2 .

12. Скарин В.Д. О методе регуляризации для противоречивых задач выпуклого программирования // Изв. вузов. Математика. 1995. № 12. Р. 81-88.

13. Popov L.D. On Accuracy estimates for one regularization method in linear programming // Optimization Problems and Their Applications (OPTA 2018) / eds. A. Eremeev, M. Khachay, Y. Kochetov, P. Pardalos. Cham: Springer, 2018. P. 170-182. (Communications Comp. Inform. Sci.; vol. 871). doi: 10.1007/978-3-319-93800-4_14.

14. Guddat J. Stability in convex quadratic programming // Mathematische Operationsforschung und Statistik. 1976. Vol. 8, no. 2. P. 223-245. doi: 10.1080/02331887608801291.

15. Dorn W.S. Duality in quadratic programming // Quart. Appl. Math. 1960. Vol. 18, no. 2. P. 407-413. doi: $10.1090 /$ qam/112751.

16. Hoffman A. J. On approximate solutions of systems of linear inequalities // J. Res. Nat. Bur. Standards. 1952. Vol. 49, no. 4. P. 263-265. doi: 10.1142/9789812796936_0018.

Поступила 19.09.2018

После доработки 21.12.2018

Принята к публикации 24.12.2018

Попов Леонид Денисович

д-р физ.-мат. наук, ведущий науч. сотрудник

Институт математики и механики им. Н. Н. Красовского УрО РАН;

профессор кафедры ВKТ

Уральский федеральный университет

г. Екатеринбург

e-mail: popld@imm.uran.ru

\section{REFERENCES}

1. Tikhonov A.N., Arsenin V.Ya. Methods for solutions of ill-posed problems. N Y: Wiley, 1981, 258 p. ISBN: 0470991240 . Original Russian text published in Tikhonov A.N., Arsenin V.Ya. Metody resheniya nekorrektnykh zadach. Moscow: Nauka Publ., 1979, 285 p.

2. Vasil'ev F.P. Metody optimizatsii [Optimization Methods]. Moscow: MTsNMO Publ., 2011, vol. 1: 620 p. ISBN: 978-5-94057-707-2 ; vol. 2: 433 p. ISBN: 978-5-94057-708-9.

3. Rockafellar R.T. Augmented Lagrange multiplier functions and duality in nonconvex programming. SIAM J. Contr., 1974, vol. 12, no. 2, pp. 268-285. doi: 10.1137/0312021. 
4. Evtushenko Yu.G. Numerical optimization techniques. N Y: Springer-Verlag, 1985, 562 p. ISBN: 978-1-4612-9530-3 .

5. Gol'shtein E.G., Tret'yakov N.V. Modified Lagrangians and monotone maps in optimization. New York: Wiley. 1996, 438 p. ISBN: 0471548219. Original Russian text published in Gol'shtein E.G., Tret'yakov N.V. Modifitsirovannye funktsii Lagranzha. Teoriya i metody optimizatsii. Moscow: Nauka Publ., 1989, $400 \mathrm{p}$.

6. Roos C., Terlaky T., Vial J.-Ph. Theory and algorithms for linear optimization: An interior point approach. N Y: Wiley, 1997, 454 p. ISBN: 0471956767.

7. Eremin I.I. Dvojstvennost'v linejnoj optimizacii (Duality theory in linear optimization). Ekaterinburg: UrO RAN Publ., 2001, 180 p. ISBN: 5-7691-1131-3.

8. Eremin I.I., Mazurov V.D., Astaf'ev N.N. Nesobstvennye zadachi lineinogo i vypuklogo programmirovaniya [Improper Problems of Linear and Convex Programming]. Moscow: Nauka Publ., 1983, 336 p.

9. Eremin I.I. Theory of linear optimization. Inverse and ill-posed problems series. Utrecht; Boston; Koln; Tokyo: VSP, 2002, 248 p. ISBN: 906764353X.

10. Skarin V.D. On the optimal correction of inconsistent problems of convex programming. Tr. Inst. Mat. Mekh., 2013, vol. 19, no. 2, pp. 267-274. (in Russian)

11. Skarin V.D. Regularization of the min-max problems occurring in convex programming. Comput. Math. Math. Phys., 1977, vol. 17, no. 6, pp. 65-78. doi: 10.1016/0041-5553(77)90173-2.

12. Skarin V.D. On method of regularization for infeasible problems of convex programming. Russian Math. (Iz. VUZ), 1995, vol. 39, no. 12, pp. 78-85.

13. Popov L.D. On Accuracy estimates for one regularization method in linear programming. In: A. Eremeev, M. Khachay, Y. Kochetov, P. Pardalos (eds.) Optimization Problems and Their Applications (OPTA 2018), Communications in Computer and Information Science, vol. 871, Cham, Springer, 2018, pp. 170-182. doi: 10.1007/978-3-319-93800-4_14.

14. Guddat J. Stability in Convex Quadratic Programming. Mathematische Operationsforschung und Statistik, 1976, vol. 8, no. 2, pp. 223-245. doi: 10.1080/02331887608801291.

15. Dorn W.S. Duality in quadratic programming. Quart. Appl. Math., 1960, vol. 18, no. 2, pp. $155-162$. doi: $10.1090 /$ qam/112751.

16. Hoffman A.J. On approximate solutions of systems of linear inequalities. J. Res. Nat. Bur. Standards, 1952, vol. 49, no. 4, pp. 263-265. doi: 10.1142/9789812796936_0018.

Received September 19, 2018

Revised December 21, 2018

Accepted December 24, 2018

Funding Agency: This work was supported by the Russian Science Foundation (project no. 1411-00109).

Leonid Denisovich Popov, Dr. Phys.-Math. Sci., Krasovskii Institute of Mathematics and Mechanics of the Ural Branch of the Russian Academy of Sciences, Yekaterinburg, 620108 Russia; Ural Federal University, Yekaterinburg, 620002 Russia, e-mail: popld@imm.uran.ru . 